\title{
Modelo de gestión de relaciones \\ con los clientes en empresas de consultoría
}

Management model of relations with
the clients in consulting companies

Efraín J. De La Hoz Granadillo

Universidad de Cartagena, Colombia.

Correo electronico:

edelahozg@unicartagena.edu.co

Ludys C. López Polo

Universidad Simón Bolivar, Colombia.

Correo electronico:

lulopez@unisimónbolívar.edu.co

Leidy Pérez Coronell

Autor de correspondencia

Universidad Simón Bolívar, Colombia.

Correo electronico:

leidy.perez@unisimonbolivar.edu.co

Información del artículo: recibido: 24 de Marzo 2017, aceptado: 10 de Julio de 2017 https://10.17081/invinno.5.2.2756 


\section{Resumen}

En este artículo de investigación se presentan los resultados obtenidos durante el desarrollo del proyecto Diseño de un modelo de Gestión de Relaciones con los clientes en una Empresa de Consultoría de Barranquilla, Colombia. Inicialmente se hace una revisión bibliográfica de los desarrollos conceptuales de la gestión de clientes, gerencia del servicio, calidad del servicio, servicio y servicio al cliente, así como de modelos relacionados con la gestión y servicio al cliente, los cuales sirvieron de soporte conceptual para el diseño del modelo propuesto. Lo anterior a través de un análisis cualitativo, descriptivo propositivo y cuantitativo, soportado en un análisis de simulación de la satisfacción de los clientes de la empresa para lo cual se utilizó la dinámica de sistemas.

\section{Palabras} Claves:

Gestión de relaciones, servicio al cliente, calidad del servicio, simulación, dinámica de sistemas. Del modelo de gestión de relaciones con los clientes, se puede concluir que mejora el nivel de satisfacción de los clientes, lo que redunda en una mayor fidelización del mismo. Finalmente el trabajo realizado constituye un aporte de importancia para el sector de consultoría en la medida que se proporciona un modelo de gestión que mejora los niveles de satisfacción de sus clientes. 


\section{Abstract}

This research paper presents the results obtained during the development of a model project Design Management Customer relations consulting firm in Barranquilla, Colombia. Initially we review the literature on the conceptual developments of Keywords: customer management, service management, service quality, and customer service,

Relationship management, customer service, quality of service, simulation, system dynamics. as well as related models and customer service management, which served to support conceptual the design of the proposed model. This through a qualitative analysis and quantitative descriptive purposeful, supported on a simulation analysis of the satisfaction of the customers of the company for which they used the system dynamics. Management model customer relationship, it can be concluded that improving the level of customer satisfaction resulting in greater loyalty itself. Finally the work is an important contribution to the field of consulting is provided as a management model that improves the levels of customer satisfaction. 


\section{Introducción}

La gestión de las relaciones con los clientes CRM (customer relationship management), no es un tema nuevo en la gerencia. Peter Drucker, en 1954 escribió "el verdadero negocio de cualquier compañía es crear y mantener a sus clientes" [1] pero este concepto cobra importancia porque las empresas son conscientes de que cada vez resulta menos rentable las estrategias dirigidas a mercados masivos debido a la demanda servicios casi personalizados.

Bajo este escenario, las empresas han redireccionado sus estrategias pasando de un enfoque tradicional (encausado hacia el producto), hacia uno con el que se logre una ventaja competitiva sostenible en el tiempo; como es el orientado hacia la relación y generación del valor al cliente. Por lo anterior, es importante analizar los procesos constituyen las bases fundamentales al momento de emprender acciones de mejora en cualquier tipo de empresas [2].

En el presente artículo de investigación, se expone un modelo de CRM en una empresa de consultoría de la ciudad de Barranquilla-Colombia, desde la perspectiva de gestión de clientes. De la revisión bibliográfica se identifican desarrollos conceptuales de la gestión de clientes, gerencia del servicio, calidad del servicio, servicio y servicio al cliente, así como de modelos relacionados con la gestión y servicio al cliente, los cuales sirvieron de soporte para el diseño del modelo conceptual propuesto. También se constató que la mayoría de las investigaciones en la materia están enfocadas a empresas de servicios diferentes a las de consultoría. De allí surge la necesidad de desarrollar un modelo que apoye la gestión de clientes en las empresas del sector de consultoría. 


\section{Revisión de literatura}

De acuerdo con Domínguez [3], gerenciar el servicio consiste en propender por la calidad de todos los momentos de verdad, de manera que exista una cultura organizacional orientada hacia el cliente, con sistemas amables y una estructura que busque colocar al cliente en primer lugar. Este "momento de verdad" es para la organización, la oportunidad para mostrar su cultura de apoyo y dedicación a sus clientes [4].

En consecuencia, cada empleado del servicio es un gerente, en alguna forma, cada uno controla el resultado del momento de verdad, ejerciendo control sobre su propio comportamiento hacia el cliente. Si el servicio no se ajusta al requerimiento del cliente, los momentos de verdad quedan arruinados y pueden conducir al descontento, merma de la lealtad y posible pérdida por completo del negocio con el cliente. Por el contrario, el servicio responde o superan las expectativas de los cliente, los momentos de verdad brillan y el cliente tiende a generalizar esas experiencias para toda la imagen del servicio [5].

Asímismo, como lo expresa Pérez [6], la conexión entre la estrategia de servicio con los sistemas sugiere que el diseño y despliegue de los sistemas físicos y administrativos se debe deducir de la definición de la estrategia de servicio. La relación estrategia del servicio y gente propone que las personas que prestan el servicio necesitan disfrutar de una filosofía calidad definida por la dirección. Finalmente, la línea de conexión gente sistemas sugiere que todas las personas de la organización, desde la alta dirección hasta los empleados de contacto con el cliente, deben trabajar dentro de los sistemas que establecen la forma de dirigir el negocio.

Por otra parte, la calidad se puede definir como el conjunto de atributos de un producto o servicio que proporcionan valor por su capacidad para satisfacer las necesidades de los clientes [7]. En esta definición se observa claramente una orientación centrada en el cliente, los cuales tienen una serie de necesidades, requisitos y expectativas. 
Deacuerdocon Mendoza [8], seentiende por serviciouna estrategia de diferenciación que se basa en el manejo de las relaciones con los clientes para la creación de valor y se fundamenta en los atributos intangibles aportados por la empresa.

En el servicio, se necesita escuchar de una manera continua a los clientes, en especial sus quejas [8].

En conclusión, como lo expresa Alcaide [9], todo esfuerzo de fidelización de clientes tiene como base fundamental, el desarrollo de una cultura orientada al cliente, la calidad del servicio que debe ser ofrecida bajo unos altos estándares y una gestión de las relaciones clara y bien estructurada que sea el centro de las estrategias de la organización. 


\section{Gestión de clientes}

La gestión de clientes es considerada como un aspecto esencial del CRM o marketing relacional como se usa en España. En este, es importante disponer de un sistema de información que cuente los elementos para anticiparse a las necesidades de los clientes; planificar los componentes de la base de datos, e implicar a todos los departamentos y empleados de la organización [10]. En este punto es importante .

Un factor fundamental para garantizar la rentabilidad en las relaciones es la gestión adecuada de las mismas mediante el seguimiento y control de indicadores para la toma de decisiones. De esta manera, la gestión de clientes se convierte en un elemento importante porque permite determinar y evaluar sus hábitos de consumo y compra, lo que es indispensable en la gestión de clientes [11].

Asimismo, la clave para el buen manejo de las relaciones con los clientes está en la capacidad que tenga la empresa para estar en continua investigación de la opinión, reclamos, pensamientos y experiencias de los clientes respecto al producto o servicio [12].

Igualmente, Guijarro [13] plantea la gestión de clientes como las estrategias de negocio, actitudes con empleados y clientes, apoyados por determinados procesos y sistemas. Se deben construir relaciones entre clientes y empresa que permitan comprender las necesidades y preferencias de cada individuo para añadir valor a productos y servicio.

De acuerdo con Miranda, Chamorro y Rubio [14], una buena gestión de las relaciones con los clientes se basa en el compromiso con los clientes, normas estándar de servicio orientadas al cliente, formación y delegación de autoridad y gestión efectiva de las quejas. Con lo cual se logra mejores resultados en los momentos de verdad de la organización. Así mismo los autores [14], consideran otras tres vías adicionales para construir la relación estable: Añadir beneficios financieros, añadir beneficios sociales y creación de dependencias estructurales. 
La gestión de relaciones con los clientes puede considerarse como el proceso mediante el cual, se desarollan y aplican estudios de mercado para construir y consolidar relaciones con la cartera que maximicen el beneficio" [15]. Las claves de este proceso están relacionadas con la capacidad de la empresa de detectar y responder a las necesidades y preferencias de los clientes en cada momento a través de la generación e integración información concreta de clientes específicos.

En resumen Barquero y Barquero [16], afirman que la gestión de clientes tiene un efecto multiplicador al ser los clientes factor de comunicación en el mercado.

De la Hoz y López [17] establecen que el manejo adecuado de la información, proporciona mejor desempeño en las organizaciones, al lograr identificar mejores oportunidades para satisfacer el cliente objetivo.

\section{Administración de relaciones con los clientes CRM}

Este es uno de los conceptos recogidos y utilizados por el mercadeo relacional para que las empresas se mantengan vigentes en el mercado, basado en el gerenciamiento de las relaciones con los clientes.

Según los autores [18], desde una perspectiva relacional, las empresas utilizan distintas estrategias para identificar a los clientes individuales, construir sólidas relaciones con ellos, facilitar la venta cruzada y la venta inducida generando valor para ambos, empresa y cliente.

En la Figura 1, se muestran los principales aspectos que han impulsado el protagonismo de la filosofía empresarial que subyace tras las siglas CRM 
enfatizando un aspecto clave, la relación simbiótica entre empresa y clientes, en la medida en que dicha estrategia incrementa el valor para ambas partes. Por su parte Régio [19] establece además una relación triada entre empresa, clientes y la fuerza de venta automatizada, con lo que se establece que el CRM proporciona en la empresa servicios distintivos para sus clientes, y relaciones personalizadas e interactivas que generan seguridad y credibilidad [20].

Sin embargo, el gerenciamiento efectivo de las relaciones con los clientes implica la optimización del manejo de la información para potenciar las estrategias de consecución de clientes, penetración y mantenimiento de sus portafolios.

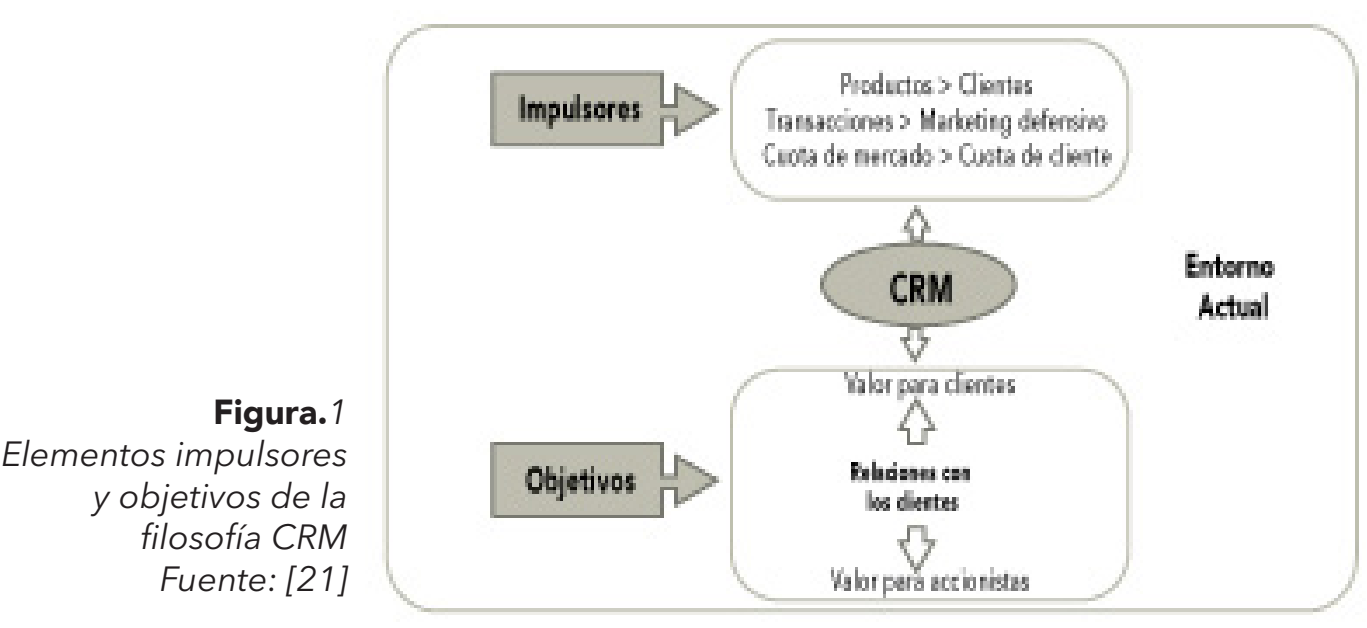


El CRM hace referencia a la estrategia de negocio focalizada al conocimiento de las necesidades del cliente, mediante el análisis de la información apoyado en la aplicación de las tecnologías de la información. de manera que se aumente el grado de satisfacción, fidelidad el cliente, es un proceso con el que puede gestionarse estratégicamente al cliente. Para ello, dicho proceso, integra diferentes dimensiones de la información relacionada con el cliente. Estas son: operaciones de contacto con el consumidor, colaboración interna entre oficinas, colaboración externa, representantes del cliente, gestión de la performance de venta y análisis de mercado y análisis de encuestas de opinión del cliente [22].

Desde la perspectiva de este autor, CRM permite a la empresa desarrollar y gestionar el conocimiento de sus clientes, en relación a procesos de contacto y servicio, para identificar los cambios en el mercado a partir del cual generar oferta de valor.

Sin embargo, antes de considerar la adopción de una herramienta de CRM, es necesario evaluar si la organización ya está orientada por una filosofía de CRM, es decir, si la empresa está centrada en el cliente, y si su cultura y la historia muestran que los esfuerzos colectivos se han hecho para crear y apoyar a largo plazo las relaciones con los clientes. Además, es necesario tener en cuenta que aunque la compañía ya está centrada en el cliente y tiene una filosofía de CRM, una herramienta de CRM aislada sin el proceso de revisión, las adaptaciones y la participación de todas las áreas y los gerentes no serán eficientes [23].

Según Drebes y Zanela [23], también es importante destacar que la comprensión del significado de la relación es una condición esencial para el desarrollo de una filosofía de CRM que tiene que guiar las estrategias de CRM o, al menos, impulsar el uso de las Tecnologías de la Información para apoyar las relaciones con los clientes. Una filosofía de CRM implica un alto nivel de compromiso, coordinación e integración entre todos los miembros de una organización y más allá, involucrando incluso a los otros actores de la cadena de valor, hacia una cultura orientada hacia el cliente. 
Pero el CRM no puede equipararse con aplicaciones tecnológicas, es un concepto estratégico, no tecnológico, que de acuerdo con Reinares [24], se apoya en tres pilares: Tecnología, procesos y recursos Humanos., los cuales son factores fundamentales del éxito o del fracaso del sistema de relaciones.

De esta manera, el surgimiento de la estrategia de negocio basada en la gestión de relaciones con clientes, es producto de la convergencia entre la orientación al mercado y el marketing relacional. En este sentido autores como Kohli \& Jaworski [25], resaltan la orientación al mercado como factor de determinante en el desempeño de la empresa independiente a la turbulencia del mercado, la intensidad competitiva o la turbulencia tecnológica del entorno. Los mismos autores reconocen que la orientación al mercado en las organizaciones contribuye con el objetivo de alcanzar ventajas competitivas de largo plazo [26]. Esta estrategia de negocio está focalizada en el cliente y la gestión integrada de la relación con él se presenta como una estrategia básica de supervivencia y crecimiento [13].

\section{Servicio al cliente}

La satisfacción en el servicio al cliente es un factor crítico para el éxito de las empresas en un mercado altamente competitivo [27].

Autores como [28], asocian el servicio al cliente con la percepción del cliente de la calidad del servicio en el que Paulrajan \& Rajkumar [29], establecen que la percepción del servicio al cliente es muy diversa, particularmente desde la perspectiva del marketing está asociado con la comunicación, precio, servicio, función, o el rendimiento de un producto. 
Como resultado de la investigación se identificaron dos tipos de modelos relacionados con la gestión estratégica de clientes. El primero corresponde a los Modelos de Calidad de Servicio, los cuales se centran en los clientes y definen la calidad del servicio en función de la percepción y las expectativas que tiene el cliente sobre el servicio. En la Tabla 1, se relacionan en forma resumida las características de cada uno de los modelos de calidad de servicio contemplados.

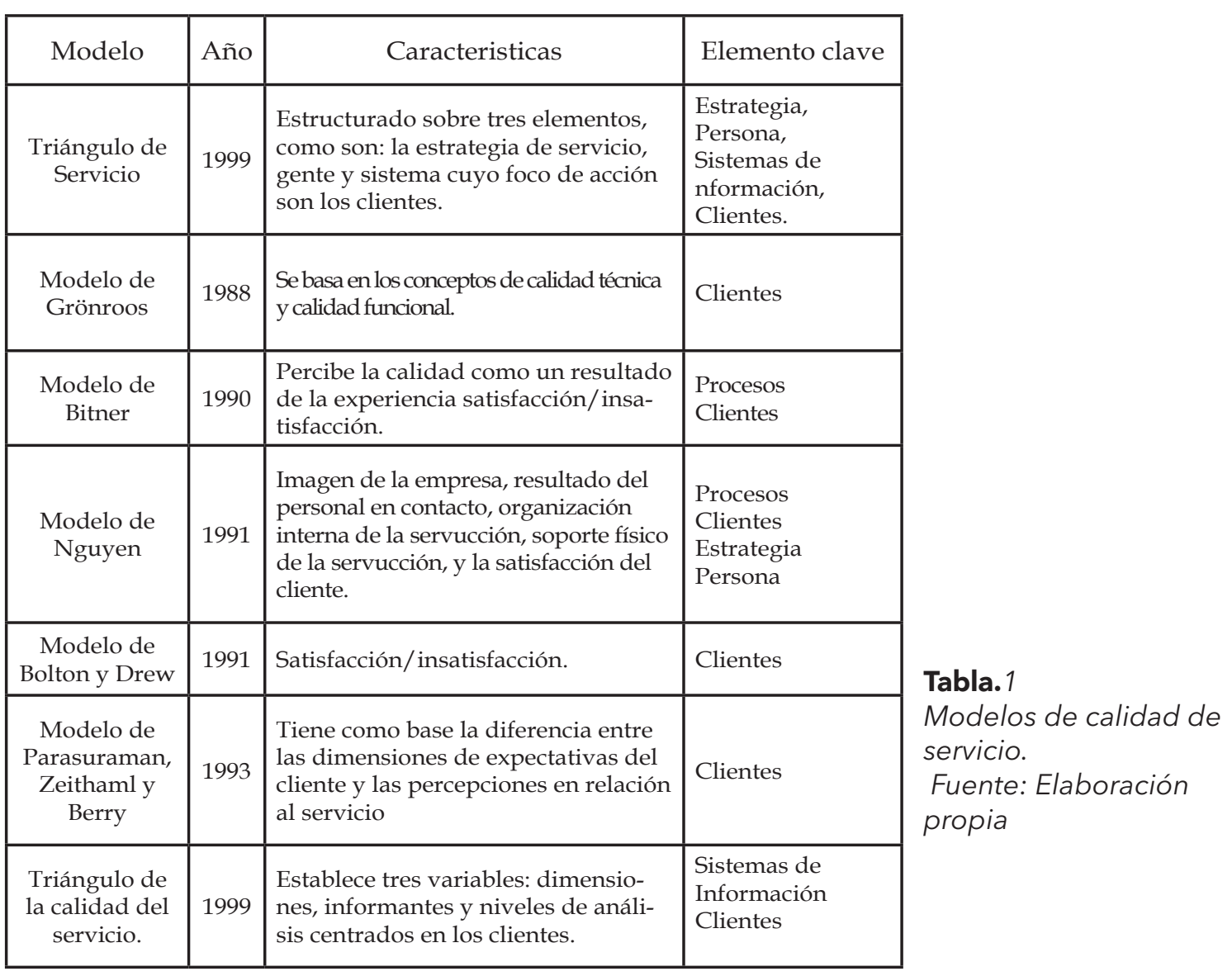


El segundo tipo corresponde a los Modelos de Gestión de Clientes. Estos se fundamentan en la relación con los clientes como factor clave de éxito y orientan la gestión de clientes a partir del tipo de relación de servicio establecido. En la Tabla 2, se muestran en forma resumida las características de cada uno de estos modelos contemplados en la presente investigación.

\begin{tabular}{|c|c|c|c|c|}
\hline & Modelo & Año & Caracteristicas & Elemento clave \\
\hline Tabla.2 & $\begin{array}{l}\text { Modelo de } \\
\text { Gestión de } \\
\text { Clientes | }\end{array}$ & 2005 & $\begin{array}{l}\text { Define dos ejes de desarrollo } \\
\text { en el primero se describen los } \\
\text { tipos de relaciones que pueden } \\
\text { desarrollarse con los clientes, y el } \\
\text { segundo establece los elementos } \\
\text { necesarios para que la gestión sea } \\
\text { realmente efectiva. }\end{array}$ & $\begin{array}{l}\text { Clientes } \\
\text { Procesos }\end{array}$ \\
\hline $\begin{array}{r}\text { de gestión de clientes } \\
\text { Fuente: Elaboración } \\
\text { propia }\end{array}$ & $\begin{array}{l}\text { Modelo de } \\
\text { las } 6 \mathrm{R}\end{array}$ & 2006 & $\begin{array}{l}\text { Define } 6 \text { factores a saber: relación, } \\
\text { retención, rentabilización, referen- } \\
\text { ciación, recuperación y reactivación, } \\
\text { los cuales pueden ser de ayuda para } \\
\text { que una relación con los clientes } \\
\text { bien gestionada se constituya en una } \\
\text { clave del éxito de las empresas. }\end{array}$ & $\begin{array}{l}\text { Procesos } \\
\text { Estrategias } \\
\text { Clientes }\end{array}$ \\
\hline
\end{tabular}

A partir del análisis de los modelos de calidad de servicio y de gestión de clientes, se identifican características claves alineadas en cinco elementos que constituyen la estructura conceptual del modelo propuesto, a saber: Estrategia, Procesos, Personas, Sistemas de Información y Cliente, los cuales se enmarcan dentro de la Organización. 


\section{Metodología}

La metodología utilizada en la investigación corresponde a un análisis cualitativo, descriptivo propositivo y cuantitativo, soportada en un análisis de la satisfacción de los clientes de la empresa. Adicionalmente, se utilizó el método inductivo, dado que se estudiaron los diferentes procesos y procedimientos que se realizan en la empresa para luego obtener un elemento general que permita establecer un modelo de gestión de clientes que abarque dichos procesos y procedimientos.

De igual forma, en la investigación se utilizó el método analítico, identificándose procesos y procedimientos, para así observar su interrelación, conocerlos, analizar por qué se realizan y el efecto que estos tienen en el logro de una gestión eficaz de la empresa.

Para la investigación se realizó un censo poblacional constituido por las 20 empresas que constituyen los clientes activos de la empresa de consultoría. Como resultado de la aplicación de los instrumentos se obtuvieron registros de 17 empresas, las tres restantes no suministraron información alguna, por lo que el estudio se limitó al número de empresas que respondieron el instrumento.

En primera instancia se hace una revisión bibliográfica de los desarrollos conceptuales de la gestión de clientes, gerencia del servicio, calidad del servicio, servicio y servicio al cliente, así como de modelos de gestión relacionados con la gestión y servicio al cliente, los cuales sirvieron de soporte conceptual para el diseño del modelo propuesto. Seguidamente se hace un análisis de los modelos relacionados con la gestión y servicio al cliente, lo que permitió definir los elementos claves en la gestión de relaciones con los clientes, elementos que sirvieron para establecer la estructura del modelo conceptual de relación con el cliente propuesto.

Una vez definido el modelo se realizó una validación del mismo, mediante una simulación utilizando la dinámica de sistemas para observar los cambios en el nivel de satisfacción de los clientes como resultado de la implementación del modelo en la organización. Esta herramienta de simulación permitió representar el estado actual de dicha organización, proyectar los resultados de las variables 
clave hacia el futuro y simular los efectos en indicadores clave en un período de valoración establecido en 52 semanas.

Para ello, se utilizó el software Vensim®PLE for Windows 6.0.0.1. En la instrumentación del modelo propuesto, se revisaron los aspectos fundamentales de cada variable con el equipo gerencial de la firma, y en función de la información recabada a través de entrevistas y de encuestas, se desarrollaron las métricas necesarias para la implementación y validación. 


\section{Modelo estratégico de gestión de relaciones con los clientes}

\section{Estructura del modelo}

A partir de los referentes teóricos y conceptuales se definió una estructura de gestión estratégica de clientes que estableciera los componentes fundamentales del modelo de gestión de relaciones con los clientes, así como la función que cumplen y la manera cómo interactúan para el flujo de la información y de los procesos operativos que conlleven a la satisfacción de los clientes y permitan a la empresa tomar las acciones encaminadas a afianzar y mejorar constantemente su posicionamiento en el mercado y entre sus clientes.

En la Figura 2 se muestra la estructura del modelo planteado y se definen a continuación las funciones de cada uno de los componentes del modelo.

Organización: Es la unidad compuesta principalmente por la Alta Gerencia y los Consultores que actúan e interactúan bajo una estructura articulada y diseñada para coordinar los recursos humanos, financieros, físicos y de información. El modelo propuesto se enmarca en la organización y se compone de cuatro elementos claves para la gestión efectiva de los clientes.

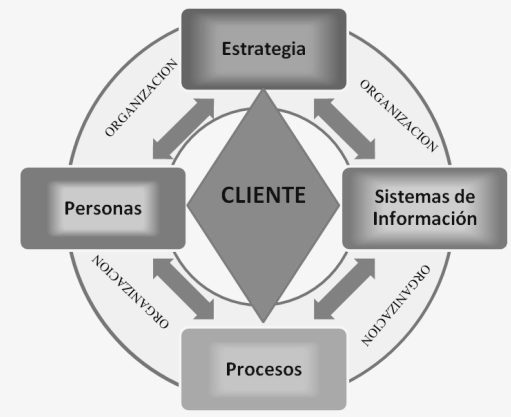

Figura 2

Modelo de gestión de relaciones con los clientes propuesto para la empresa de consultoría. Fuente: Elaboración de los autores, 2012 
La Alta Gerencia se encarga del direccionamiento de la empresa y de la definición de todas las estrategias necesarias para cumplir con su misión y visión. Se encarga de establecer los indicadores de gestión de los clientes con los que se evalúan los resultados de la organización y establece la ruta organizacional que debe seguir la entidad para el logro de sus objetivos misionales.

Los Consultores son los encargados de operacionalizar las estrategias de la organización. Además, son quienes generalmente establecen un primer contacto con los clientes. Trabajan directamente con la Alta Gerencia retroalimentando el proceso de captación de necesidades y expectativas de los clientes, insumo necesario para el diseño de un portafolio de servicios innovador. Participan en la construcción de indicadores de gestión y tienen siempre contacto directo con el cliente, lo que los hace un actor importante del proceso de gestión orientado hacia su fidelización.

Clientes: Son la figura central del modelo, la razón de ser de la empresa, puesto que es la fuente de ingresos y rendimientos. Representa el foco de acción de las estrategias dirigidas hacia su conocimiento en profundidad para captar sus necesidades y expectativas. Es quien demanda servicios diferenciadores con valor agregado para establecer relaciones a largo plazo con la empresa.

Persona: Se define como el recurso humano que trabaja en la organización, quien interactúa directa o indirectamente con los clientes y constituye la primera línea de contacto con éstos, tanto para la prestación del servicio como para la identificación de necesidades y oportunidades de mejora en la satisfacción de los clientes. Sin embargo, para garantizar la fidelización de los clientes es necesario el respaldo de la Alta Gerencia y el apoyo de una estrategia de orientación al cliente. 
Sistemas de Información: Corresponden a la estructura definida para el flujo de información en doble sentido cliente-organización, así como al interior de la organización a través de cada uno de sus elementos fundamentales. Los sistemas de información garantizan que se disponga de información suficiente y confiable para el proceso de toma de decisiones, permite conocer las expectativas de servicio y evaluar la percepción que tiene cada cliente del mismo. En este punto se incluye toda la plataforma tecnológica necesaria para asegurar que la información pertinente esté disponible cuando se requiera.

Estrategias: Definen los objetivos generales de la empresa y los cursos de acción fundamentales, de acuerdo con los medios actuales y potenciales de la empresa, a fin de alcanzarlos y posicionar competitivamente a la organización en su entorno. En este sentido las estrategias definen las acciones encaminadas a superar las expectativas de servicio del cliente respecto a la organización. Las estrategias son construidas con base en la información que se tenga de los clientes y tiene dos funciones, una de ellas es la interna que se centra en el cliente interno y la externa que se enfoca en el cliente externo.

Procesos: Los procesos los constituyen el conjunto de actividades secuenciales que desarrolla la organización y que tienen por objetivo generar unos resultados acordes a lo planificado. En el Modelo Estratégico de Gestión de Clientes, los procesos direccionan la correcta implementación de las estrategias y las acciones ejecutadas por el personal, así como el eficiente y efectivo aprovechamiento de los sistemas de información.

Tiene como objetivo conocer y entender profundamente a los clientes a través de la ejecución de unas estrategias apoyadas en el flujo permanente de información con el fin de captar efectivamente sus necesidades y expectativas y se pueda ofrecer un servicio adecuado y diferenciado, que se perciba "casi personalizado" y que en consecuencia facilite la retención de los clientes. Este proceso que hace parte del eje estratégico de la Organización aumentaría el control de la relación con los clientes, mejoraría la percepción de los clientes frente al conocimiento de sus necesidades y disminuiría la tasa de abandono de los clientes. La ejecución 
de este proceso alimenta la capacidad predictiva y reactiva de la empresa para atender adecuadamente a cada uno de sus clientes, facilitándose la construcción de relaciones duraderas entre éstos y la Organización.

\section{Funcionamiento del modelo}

Cliente-Organización: El proceso parte de las necesidades y expectativas identificadas en los clientes, las cuales representan un insumo para la empresa y son captadas por la organización compuesta por la alta gerencia y el grupo de consultores. En esta parte es fundamental disponer de instrumentos de evaluación de cada uno de los momentos de verdad que tiene la organización con sus clientes, del análisis de las evaluaciones se deben identificar debilidades y fortalezas en los procesos de consultoría, así como oportunidades y amenazas en la fidelización de los clientes.

Organización-Estrategias: La gerencia establece las políticas y junto con los consultores determinan las estrategias de gestión encaminadas a fortalecer la fidelización del cliente. Es importante que la organización defina como resultado de la evaluación de sus momentos de verdad, quejas, sugerencias y reclamos posteriores al servicio, cuáles serán las estrategias para afianzar fortalezas y remover o minimizar debilidades, así como el aprovechamiento de las oportunidades que brinda el mercado de clientes, sin dejar de lado aquellos factores externos que pueden llevar a perder un cliente (estrategias de la competencia). Una vez establecidas las 
estrategias de gestión, se realiza seguimiento del impacto que éstas tienen en el cliente, para realizar los ajustes necesarios.

Estrategias-Personal: Es fundamental que tanto directivos, como consultores y personal auxiliar de la empresa conozcan las estrategias de gestión encaminadas al aseguramiento de la satisfacción del cliente y se sienta empoderado para contribuir en el desarrollo de las estrategias. En esta parte se requiere de capacitación continua de los procesos y estrategias diseñadas.

Personal-Procesos: El diseño de los procesos, debe estar orientado a facilitar el acompañamiento de consultoría en los clientes, respondiendo a expectativas y siendo eficaces en la consecución de resultados.

Procesos-Sistemas de Información: El modelo está soportado en un proceso continuo de flujo de la información, con lo cual se requiere que esté sustentado en herramientas tecnológicas que faciliten el seguimiento y control de proceso. Asimismo, el modelo comprende el desarrollo de indicadores para la evaluación de resultados que permitan implementar ajustes en las estrategias diseñadas. 


\section{Resultados}

A partir del análisis del problema, se definieron métricas necesarias para la simulación, las cuales se describen a continuación:

Servicio Percibido: Representa el promedio de evaluación del servicio recibido. Se define como (ver Eq. 1):

Servicio Percibido $=($ Atención Necesidades Específicas Percibido + Calidad Informes Percibido +Calidad Servicio Posventa Percibido + Captación de Necesidades Percibido + Confianza y Seguridad Percibida + Conocimiento Percibido + Cortesía Percibida + Cumplimiento a Tiempo Percibido + Disposición Atención Cliente Percibido + Efectividad Servicio

Percibido+ Información Actualizada Percibida + Informes Oportunos

Percibido + Presentación Consultores Percibida + Rapidez del Servicio Percibido + Tiempo Tramite Oferta Percibido) $/ 15$

Servicio Esperado: Evalúa el nivel promedio de servicio esperado por el cliente. Se define como (ver Eq. 2):

Servicio Esperado: (Atención de Necesidades Específicas Esperada + Calidad Servicio Posventa Esperado + Captación de Necesidades Esperada + Calidad Informes Esperado + Confianza y Seguridad Esperada + Conocimiento Esperado + Cortesía Esperada + Cumplimiento a Tiempo Esperado + Disposición Atención Cliente Esperado + Efectividad Servicio

Esperada + Información Actualizada Esperada + Informes Oportunos Esperados + Presentación Consultores Esperada + Rapidez del Servicio Esperada + Tiempo Tramite Oferta Esperado)/15.

Nivel de Insatisfacción: Representa la brecha que existe entre el Servicio Esperado y el Servicio Percibido. Matemáticamente se representa como (ver Eq. 3): 
Nivel de Insatisfacción= Servicio Esperado-Nivel de Satisfacción

Tasa de Satisfacción: Comprende la rata o velocidad de flujo a la que se cierra la brecha que existe entre el Servicio Esperado y el Servicio Recibido. Constituye el mejoramiento del servicio como resultado de los procesos del Modelo de Gestión de Relaciones con los Clientes propuesto. Matemáticamente se expresa como (ver Eq. 4):

Tasa de Satisfacción = Nivel Insatisfecho/Tiempo de Revisión

Nivel de Satisfacción: Es una variable clave en la validación del modelo, puesto que permitire analizar el cierre de brecha entre el servicio percibido y el servicio esperado. Se define como (ver Eq. 5):

Nivel de Satisfacción $=$ Servicio Percibido +Tasa de satisfacción

Tiempo de Revisión: Se define como el tiempo necesario para planear e implementar los procesos de mejora del modelo. Matemáticamente se representa por los tiempos de diseño de estrategias, rediseño de proceso y capacitación del personal (ver Eq. 6).

Tiempo de Revisión $=$ Diseño Estrategias + Personal + Rediseño Procesos

Una vez definidas las métricas requeridas para la simulación se realizó el Diseño Dinámico del Sistema como se muestra en la Figuras 3 y 4 . En el modelo de Dinámica de Sistema puede observarse como interactúa el sistema de información para identificar expectativas y percepciones del servicio. Así mismo se observa como los componentes del modelo Personal, Estrategias y Procesos intervienen en el mejoramiento del servicio aumentando la tasa de satisfacción. Estos componentes del modelo abordan el diseño de estrategias, el rediseño de procesos y la capacitación del personal, los cuales establecen el tiempo de revisión durante el cual se mejora de manera continua el servicio de la organización. 


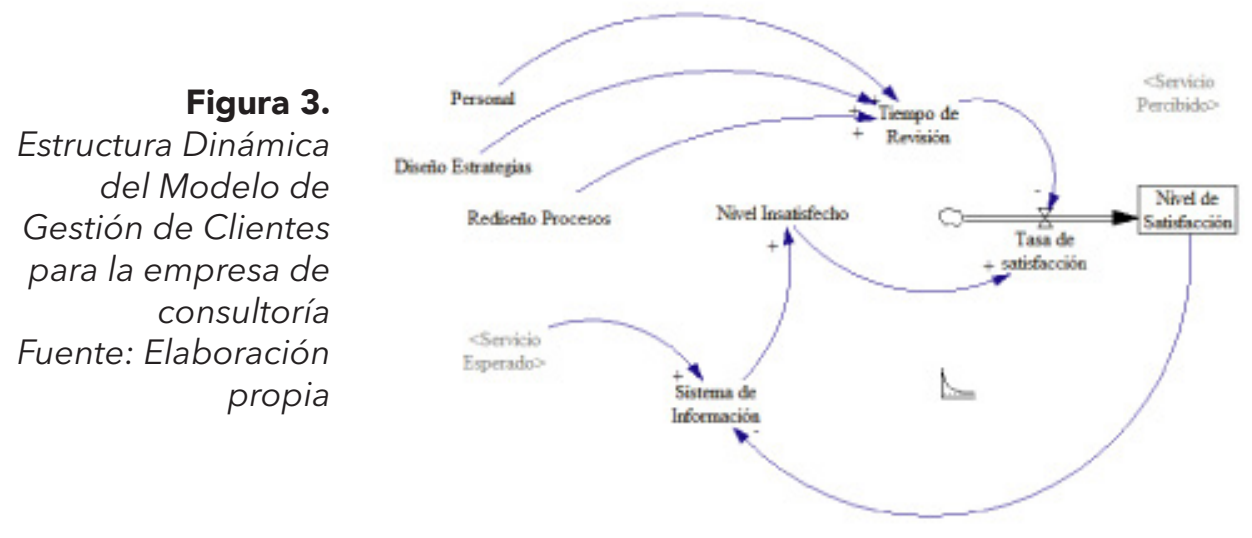

Figura 4.

Componentes de las métricas para la evaluación del servicio.

Fuente: Elaboración

propia

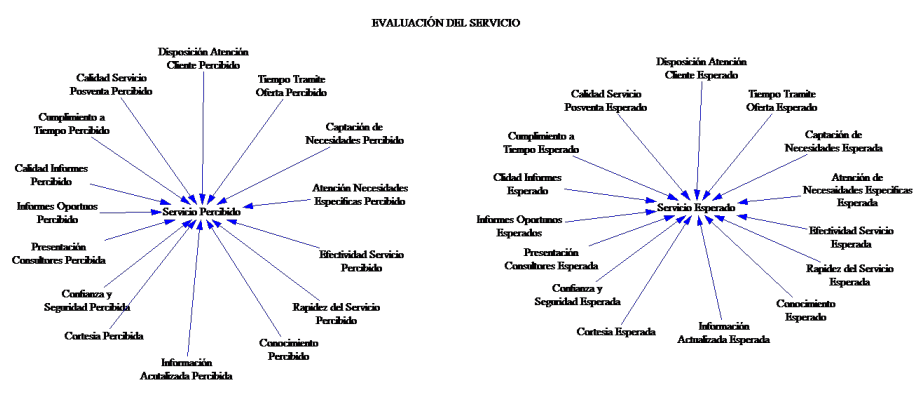

A continuación se realiza un análisis detallado del impacto del modelo en las métricas del servicio, lo cual permitirá validar el modelo.

En la Figura 5 se muestra la evolución en el Nivel de Satisfacción en un periodo de un año (52 semanas), con la cual se evidencia el mejoramiento de este factor a partir de la implementación del modelo de gestión. 


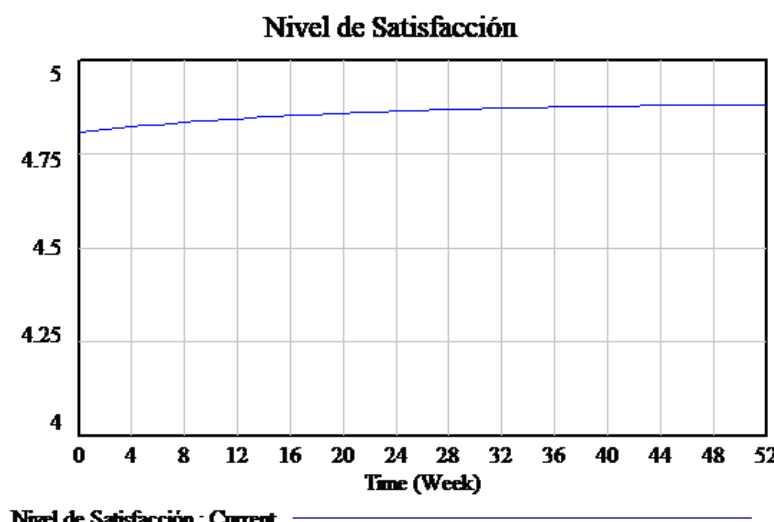

Figura 5.

. Gráfica del Nivel de Satisfacción.

Fuente: Elaboración propia

Así mismo, en la Figura 6 se muestra como la tasa de satisfacción del cliente disminuye a medida que el nivel de satisfacción se acerca a las expectativas de los clientes, por lo que se requiere de mayor esfuerzo por parte de la organización.

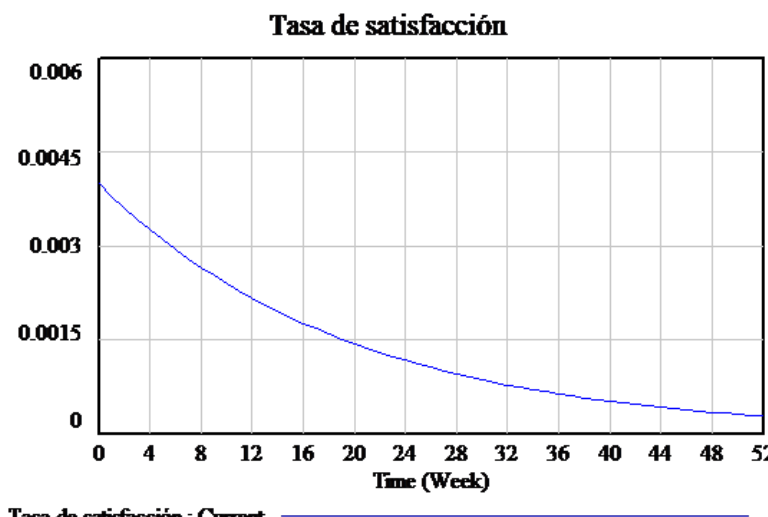

Figura 6.

Tasa de Satisfacción.

Fuente: Elaboración propia 
En la Figura 7 se observa que consecuentemente con la Figura 5, el nivel de insatisfacción disminuye rápidamente en las primeras 36 semanas, siendo mínimo en el periodo restante.

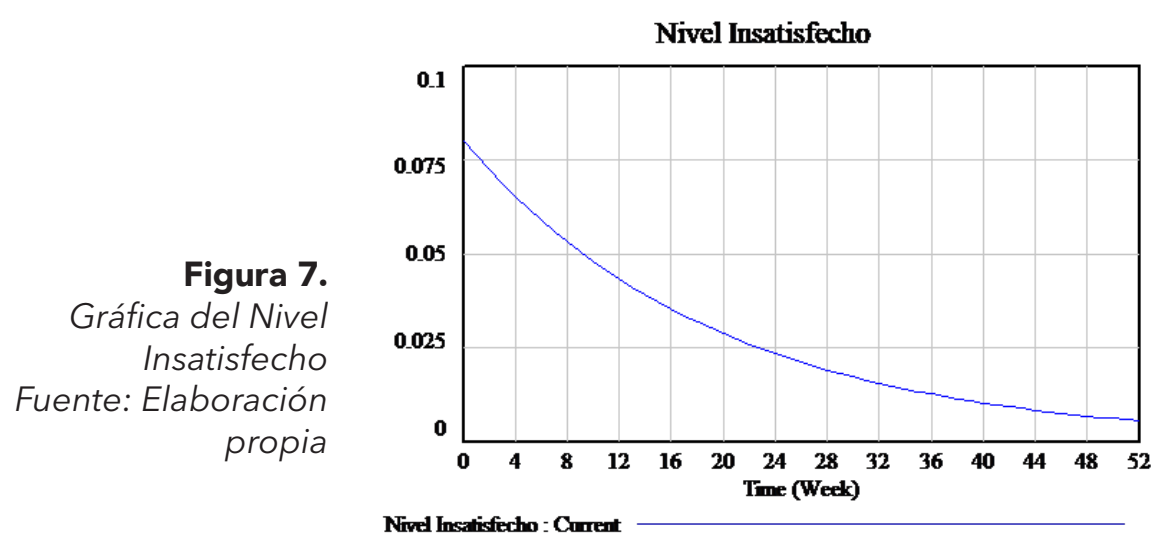




\section{Conclusión}

En el presente trabajo de investigación se planteó como objetivo proponer un modelo de gestión de relaciones con los clientes aplicado a empresas de servicio, para lo cual se hizo un análisis de gestión de relaciones con los clientes, soportado en la fundamentación teórica y examinando diferentes investigaciones sobre la temática, con lo se pudo determinar variables como los procesos, las personas, la estrategia y los sistemas de información como elementos clave de la gestión de relaciones con los clientes. Lo que sirvió para identificar que variables inciden en el proceso y así diseñar un modelo de gestión que sirva de apoyo para la toma de decisiones orientada siempre hacia la conservación de los clientes.

Partiendo de la relevancia que tienen las relaciones con los clientes en las organizaciones del sector servicios, el estudio se centró en una empresa de consultoría, examinando sus procesos, recursos y grado de relaciones para poder obtener un modelo ajustado a sus necesidades.

Así mismo, se verificó que al desarrollar y probar el modelo estratégico de gestión de relaciones con el cliente en la empresa de consultoría, la organización puede, a partir de la implementación de estrategias orientadas al cliente, mejorar su nivel de satisfacción y disminuir el grado de insatisfacción, acercándose al nivel del servicio esperado por el cliente. Es importante tener en cuenta que dichas estrategias deben focalizarse en los aspectos identificados como más débiles y susceptibles de mejora, tales como "mecanismos para captar las necesidades del cliente" y "rapidez en la prestación del servicio", sin dejar a un lado las estrategias que continúen afianzando los factores claves de éxito, identificados como fortalezas actuales de la empresa: "Conocimiento para responder a las consultas del cliente" y "Disposición para la atención al cliente".

El modelo para la gestión de las relaciones con el cliente propuesto, se apoya en una estructura organizacional orientada al cliente que facilite su satisfacción a través de la atención personalizada oportuna, soportada por un equipo dentro de unos procesos flexibles. Procesos que en cada momento, no solo brinden al cliente lo que necesita, sino que sepan anticiparse a las necesidades del mismo, para ofrecer un servicio que mejore su "cadena de valor" haciéndolos más 
competitivos. Estos elementos se articulan con los sistemas de información que retroalimentan el sistema, para garantizar que la mejora de las relaciones con el cliente redunde en una alta satisfacción y confianza del mismo.

Finalmente el CRM, constituye una estrategia de gestión de importancia para la consolidación competitiva de la organización en la medida que se establece un sistema de mejora continua, con el cual se evalúan oportunidades y amenazas del mercado.

Para lo anterior es importante que los miembros de las organizaciones quienes tienen la capacidad de pensar y de reorientar los procesos y las actividades que se ejecutan al interior de estas [30], analicen las necesidades, expectativas y deseos de sus clientes para diseñar estrategias de mercadeo orientadas precisamente para ellos [31,32]. 


\section{Referencias Bibliográficas}

1. García Varcárcel, I. (2001). CRM Gestión de la relación con los clientes. España: Fundación Confemetal.

2. T. Fontalvo, E. DeLa Hozy D. Cardona, “Diseño de un plan de mejoramiento para la cadena de suministro de la empresa Drolitoral SA, Aplicando el Modelo SCOR", Revista Soluciones de Postgrado EIA, Vol. 1 nº. 6, pp. 33-53, 2010.

3. H. Domínguez Collins, El Servicio Invisible. Bogotá: ECOE Ediciones, 2006

4. A. Nosnik Ostrowiak, Culturas Organizacionales: su origen, consolidación y desarrollo. España: Netbiblio S.L., 2005.

5. Y.Perdomo\&R.Prieto, "ElLiderazgocomoHerramienta deCompetitividad para la Gerencia del Servicio", CIGAG, vol. VI, n. 2.,2009.

6. V. Pérez Torres, Calidad Total en la Atención al Cliente (Primera ed.). España: Ideas Propias Editorial.,2006.

7. S.L. Vertice. La Calidad en el Servicio al Cliente. Malaga, España: Vertice.,2008.

8. J. Mendoza, "La relación cliente-servidor en el sistema de servicio", Desarrollo Gerencial, vol. 2, pp.41-57.,2010.

9. J, Alcaide, Fidelización de Clientes. Madrid: ESIC Editorial, 2010.

10. D. Páramo Morales, \& E. Ramírez Plazas, Gerencia Estratégica de Marketing: Un Enfoque Cultural. Bogotá: Universidad Surcolombiana, 2010.

11. J. Valencia Gaviria, "Fidelización y lealtal como estrategias para impactar favorablemente el recaudo y la cartera de las empresas", El Cuaderno Ciencias Estratégicas, pp. 11-22, 2008.

12. A. M. Gil-Lafuente, \& C. Luis-Bassa, La Innovación Centrada en el Cliente Utilizando el Modelo de Inferencias en una Estrategia CRM (Vol. 17). Barcelona: Universidad de Barcelona.,2011

13. M. Guijarro García, Estudio de la Literatura y Modelos de Negocio de la Implantación de CRM -Modelo Cliente Céntrico- como Enfoque Estratégico condicionante de la Ventaja Competitiva en la PYME: Estudio Empírico de la Aplicación de un CRM en Agencias de Viajes. Valencia,2009.

14. F. Miranda González, A. Chamorro Mera, \& S. Rubio Lacoba, Introducción a la Gestión de la Calidad. Madrid: Delta Publicaciones.,2007.

15. P. Cabanelas Lorenzo, \& A. Moreira, "La Gestión de las Relaciones con los Clientes y la Empresa de Alta Rentabilidad de Galicia", Revista de Estudios 
Regionales, vol.84, pp. 223-246.,2009.

16. J, Barquero y M, Barquero, El libro de oro de las relaciones públicas y el marketing (Quinta ed.). Barcelona: Ediciones Deusto, 2008.

17. E, De la Hoz y L, López, "Análisis conceptual del desarrollo sostenible y el desarrollo sustentable", Revista Investigación e Innovación en Ingeniería, Vol. 2, n. 2, pp. 24-28, 2014.

18. J. Dawson, A. Jiménez Zarco, M. Martínez Ruiz, \& M. Llamas Alonso, "Medición de Resultados en la Estrategia CRM: Hacia un Modelo Holístico", Boletín Económico de ICE (Información Comercial Española), pp. 35-54., 2006.

19. F. Régio, "Automação do processo de vendas no contexto do gerenciamento de clientes por CRM", Revista de Administração da Universidade Federal de Santa Maria, vol. 5, nº 1, pp. 29-46., 2012.

20. S. Toriani, \& M. Angeloni, "CRM as a support for knowledge management and customer relationship", JISTEM J.Inf.Syst. Technol. Manag. (Online) vol.8 $\mathrm{n}^{\circ} .1 ., 2011$.

21. J. Dawson, A. Jiménez Zarco, M. Martínez Ruiz \& M. Llamas Alonso, Medición de Resultados en la Estrategia CRM: Hacia un Modelo Holístico. Boletín Económico de ICE (Información Comercial Española), 35-54.,2006.

22. M, Bernárdez, Capital Intelectual: creación de valor en la sociedad del conocimiento, Blomington (E.U), 2008.

23. C. Drebes Pedron \& A. Zanela Saccol, “What lies behind the concept of customer retionship management? discussing the essence of CRM through a phenomenolgical approach", Brazilian Administration Review, vol. 6, $\mathrm{n}^{\circ}$. pp. 34-49.,2009.

24. P. Reinares, 100 Errores del CRM. Madrid: ESIC.,2009

25. A. Kohli, \& B. Jaworski, "Market Orientation: The Construct, Research Propositions", Joumal of Marketing, Vol. 54,pp. 1-18.,1990.

26. A. Kohli \& B. Jaworski, "Market Orientation: Antedents and Consequences", Joumal of Marketing, Vol. 57, pp. 53-70.,1993.

27. G. Tontini \& A. Sant'Ana, "Interação de atributos atrativos e obrigatórios de um serviço na satisfação do cliente", Prodção, vol.18 no.1 São Paulo, 2008. 
28. H. Carrasco, V. Martínez-Tur, J. Peiró, y C. Moliner, "Validation of a Measure of Service Climate in Organizations", Rev. psicol. trab.organ, vol.28 $\mathrm{n}^{\circ}$.2., 2012.

29. R. Paulrajan, \& H. Rajkumar, Service Quality and Customers preference of Cellular Mobile Service Providers. Journal of Technology Management \& Innovation. J. Technol. Manag Innov.Volume 6, Issue I, pp.38-45., 2011.

30. E, De La Hoz y L, López, “Aplicación de Técnicas de Análisis de Conglomerados y Redes Neuronales Artificiales en la Evaluación del Potencial

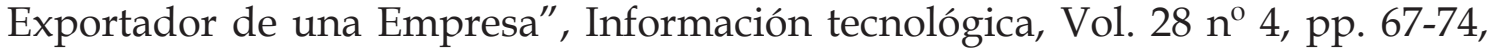
2017.

31. V. Iguaran, y L. Campo, "Eficiencia en la productividad desde la perspectiva del cliente interno y externo en las empresas recicladoras del plástico en el departamento de la Guajira-Colombia", Revista Investigación e Innovación en Ingenierias,vol. 5, $\mathrm{n}^{\circ} .1$, pp. 72-9, 2017.

32. L. Ferrer Castellanos, K. González Insignares and L. Mendoza Vega, “La innovación como factor clave para mejorar la competitividad de las pymes en el departamento del Atlántico, Colombia", Dictamen Libre, n. 16, pp. 21-36, 2015. 


\section{Este artículo se cita}

E. De La Hoz, L. Lopez., y L. Perez, "Modelo de gestión de relaciones con los clientes en empresas de consultoría",

Investigación e Innovación en Ingenierias, vol. 5, nº 2, pp. 46-77., 2017 